\title{
OPEN Diffusion tensor imaging for the study of early renal dysfunction in patients affected by bardet-biedl syndrome
}

\author{
Pasquale Borrelli ${ }^{1 凶}$, Miriam Zacchia ${ }^{2}$, Carlo Cavaliere ${ }^{1}$, Luca Basso ${ }^{1}$, Marco Salvatore ${ }^{1}$, \\ Giovambattista Capasso ${ }^{2,3}$ \& Marco Aiello ${ }^{1}$
}

Kidney structural abnormalities are common features of Bardet-Biedl syndrome (BBS) patients that lead to a progressive decline in renal function. Magnetic resonance diffusion tensor imaging (DTI) provides useful information on renal microstructures but it has not been applied to these patients. This study investigated using DTI to detect renal abnormalities in BBS patients with no overt renal dysfunction. Ten BBS subjects with estimated glomerular filtration rates over $60 \mathrm{ml} / \mathrm{min} / 1.73 \mathrm{~m}^{2}$ and 14 individuals matched for age, gender, body mass index and renal function were subjected to high-field DTI. Fractional anisotropy (FA), and mean, radial and axial diffusivity were evaluated from renal cortex and medulla. Moreover, the corticomedullary differentiation of each DTI parameter was compared between groups. Only cortical FA statistically differed between BBS patients and controls $(p=0.033)$, but all the medullary DTI parameters discriminated between the two groups with lower FA $(p<0.001)$ and axial diffusivity $(p=0.021)$ and higher mean diffusivity $(p=0.043)$ and radial diffusivity $(p<0.001)$ in BBS patients compared with controls. Corticomedullary differentiation values were significantly reduced in BBS patients. Thus, DTI is a valuable tool for investigating microstructural alterations in renal disorders when kidney functionality is preserved.

Bardet-Biedl syndrome (BBS) is a rare inherited disorder characterised by the dysfunction of several organs. To date, at least 24 genes (BBS 1-24), most encoding proteins localised within the basal body of the primary cilium $^{1}$, have been associated with human BBS. BBS patients are prone to develop renal failure ${ }^{2}$. Abnormal renal and urinary tract structures have been reported ${ }^{3,4}$; accordingly, chronic kidney disease (CKD) is considered the most common cause of morbidity and mortality. The renal phenotypes of both patients and mouse models are variable, although a tubulointerstitial defect is postulated to be a common substrate ${ }^{5}$ because of the low incidence of proteinuria and the high rate of urine concentration defects ${ }^{6,7}$.

In the last decade, magnetic resonance imaging (MRI) has been increasingly used for in vivo kidney studies. In addition to the clinical utility of MRI examinations for the assessment of several structural kidney abnormalities (e.g., autosomal dominant polycystic kidney disease ${ }^{8}$ and renal cancer ${ }^{9,10}$ ), recent studies have focused on the ability of kidney MRI to provide quantitative functional biomarkers for both diagnostic and prognostic purposes $^{11-16}$. In this field, diffusion tensor imaging (DTI) provides useful insights into in vivo kidney analyses by measuring, noninvasively, physical properties related to kidney tissue microstructural integrity ${ }^{17}$. Indeed, the water movement analysis provided with the DTI allows the measurement of quantitative parameters, such as mean diffusivity (MD) and fractional anisotropy (FA), which reflect relevant features strictly related to the functional integrity of renal structures, such as tubules, collecting ducts and vessels ${ }^{18,19}$. In addition to the characterisation of healthy kidneys ${ }^{20-22}$, DTI has been successfully applied to detect renal damage in different kidney disorders, such as CKD ${ }^{23-25}$, kidney transplant ${ }^{26-28}$, diabetic nephropathy ${ }^{29-31}$, renal clear cell carcinoma ${ }^{32}$ and glomerulonephritis ${ }^{33}$. In these contexts, the absence of a contrast media makes the DTI analysis particularly suitable for subjects with kidney failure in which contrast media are highly contraindicated.

The principal aim of the present study was to investigate how microstructural parameters derived from DTI can be used as markers of kidney impairment in patients with BBS. Specifically, we assessed the utility of DTI in detecting early kidney alterations in a subset of BBS individuals with preserved renal function. To

${ }^{1}$ IRCCS SDN, Via Emanuele Gianturco 113, 80131 Naples, Italy. ${ }^{2}$ Department of Medical and Translational Sciences, University of Campania L. Vanvitelli, Naples, Italy. ${ }^{3}$ Biogem, Research Institute for Molecular Biology and Genetics, Ariano Irpino, Italy. ${ }^{\circledR}$ email: pasquale.borrelli@synlab.it 
date, no dedicated quantitative MRI kidney studies in BBS are present in the literature. Indeed, only structural macro-abnormalities (e.g. kidney volume, cysts, malformations and fetal lobulations) have been detected by the application of MRI in BBS ${ }^{34,35}$. To the best of our knowledge, this is the first study focused on using DTI for in vivo studies on renal microstructural anomalies in BBS subjects. To achieve the present aim, DTI parameters of BBS subjects were compared with those of controls, which were matched for age, gender and renal function. Moreover, corticomedullary differentiation (CMD) obtained from DTI parameters was measured in patients and controls, and the right and left kidney were analysed separately using DTI parameters to assess possible asymmetrical alterations.

\section{Materials and methods}

Study participants. This study was approved by the local ethics committee (IRCCS Pascale) with protocol number $02 / 17$. All the procedures adopted in this study were performed in accordance with the approved guidelines and regulations. Written informed consent was obtained from all the subjects enrolled in this study. From May 2018 to August 2019, 10 BBS patients referred to the Nephrology Unit of the University of Campania L. Vanvitelli were enrolled in the study. The diagnosis was based on Beales clinical criteria ${ }^{36}$. Retinal degeneration was assessed by ophthalmologic evaluation and learning disabilities were defined as defects in writing, reading and spelling, as well as deficiencies in memory, coordination and emotional maturity. Obesity was defined as a body mass index (BMI) higher than $30 \mathrm{~kg} / \mathrm{m}^{2}$. Non-hypertensive subjects were defined as people with blood pressure $<140 / 90 \mathrm{mmHg}$ in the absence of dedicated treatment. Non-diabetic individuals were defined as those with plasma $\mathrm{HbA} 1 \mathrm{c}$ level $<7 \%$, without a personal history of diabetes mellitus. Genetic validation was available for all the enrolled BBS patients. Renal function was assessed by estimating the glomerular filtration rate using the Chronic Kidney Disease Epidemiology Collaboration (CKD-EPI) equation with standardized plasma creatinine measurements. Genetic validation was available for all the enrolled patients.

The inclusion criteria were as follows: more than 18 years old, no contraindications to MRI examinations (i.e., metal implants, claustrophobia or pregnancy status), estimated glomerular filtration rate (eGFR) greater than $60 \mathrm{ml} / \mathrm{min} / 1.73 \mathrm{~m}^{2}$.

In total, 14 subjects with no history of renal diseases, diabetes or hypertension were enrolled as the control group. The inclusion criteria of the controls were as follows: no contraindications to MRI examinations, eGRF greater than $60 \mathrm{ml} / \mathrm{min} / 1.73 \mathrm{~m}^{2}$, absence of renal pathologies, diabetes, hypertension or metabolic abnormalities. Moreover, control subjects were selected by matching the gender, age range and BMI of the BBS group members.

MRI protocol. Both BBS and control subjects underwent MRI examinations with the same acquisition protocol using a 3 T scanner (Biograph mMR; Siemens, Erlangen, Germany), equipped with a 4-channel phasedarray body coil.

Anatomical T1- and T2-weighted images, covering both the right and left kidney, were acquired to examine kidney morphology. The T1-weighted images were acquired with the 2D Fast Low Angle Shot (FLASH) sequence, whereas T2-weighted images were obtained with the half-Fourier acquisition single-shot turbo spin echo (HASTE) sequence. Both FLASH and HASTE images were acquired with a coronal orientation in breath-hold.

For DTI images, a fat-saturated twice-refocused EPI sequence was performed with a respiratory triggering, $500 \mathrm{~s} / \mathrm{mm}^{2}$ as diffusion weighting and three averages each of which with six different diffusion directions at $b=500 \mathrm{~s} / \mathrm{mm}^{2}$ and two volumes at $b=0 \mathrm{~s} / \mathrm{mm}^{2}$. A diffusion weighting of $b=500 \mathrm{~s} / \mathrm{mm}^{2}$ was chosen as a good compromise between image quality and pure diffusion measurement (i.e., by neglecting the perfusion phenomena) and considering the mean diffusivity of the kidneys as reported in the literature ${ }^{37-39}$. Additional parameters of the EPI sequence were as follows: oblique-coronal orientation, repetition time $1800 \mathrm{~ms} / \mathrm{echo}$ time $74 \mathrm{~ms} ; 152 \times 152$ matrix; $319 \times 319 \mathrm{~mm}^{2}$ field of view; slice thickness of $3 \mathrm{~mm} ; 2.1 \times 2.1 \mathrm{~mm}^{2}$ pixel size; 36 slices; 2 as the parallel imaging accelerator factor and left to right as the phase-encoding direction. Another set of DTI images was acquired with the same parameters by only blipping the phase-encoding direction (right to left) for the subsequent geometric distortion correction ${ }^{39}$. The total scan time varied from 15 to 20 min, depending on the individual's respiratory cycle.

Image analysis. Prior to image analyses, the images were de-identified and visually checked for image artefacts. In particular, the raw image data in the DICOM format were elaborated with the software provided with the scanner to remove the DICOM tags correlated with patient' data, and then, they were transferred to a workstation for the subsequent image analyses.

The acquired images were analysed by an expert radiologist (C.C.) with more than 8 years of experience in abdominal imaging. In particular, the radiologist assessed the image quality by visually checking for the absence, or low presence, of image artefacts such as gross movement, ghosting and signal loss. Moreover, both the FLASH and HASTE images were inspected to detect renal structural abnormalities (i.e. cysts or fetal lobulations).

The DTI images were pre-processed for mitigating image artefacts such as movement, eddy-currents and geometric distortion. The pre-processing procedure was chosen to provide the highest test-retest reproducibility results, as reported in Borrelli et al. ${ }^{39}$. In particular, the movement between and within the DTI series and the distortions induced by eddy-currents were corrected using the eddy command, whereas the susceptibility-induced geometric distortions were corrected using the topup function. Both dedicated routines were implemented in the FMRIB-FSL library (v. 6.0.0 ${ }^{40}$. For the geometric distortion correction, an approach based on the estimation of the susceptibility off-resonance field ${ }^{41}$ was adopted using additional DTI data acquired with the opposite phase-encoding direction.

Parametric maps derived from the pre-processed DTI images were evaluated using MRtrix 3 toolbox ${ }^{42}$. In particular, MD, FA, radial diffusivity (RD) and axial diffusivity (AD) were evaluated from the eigenvalues of 

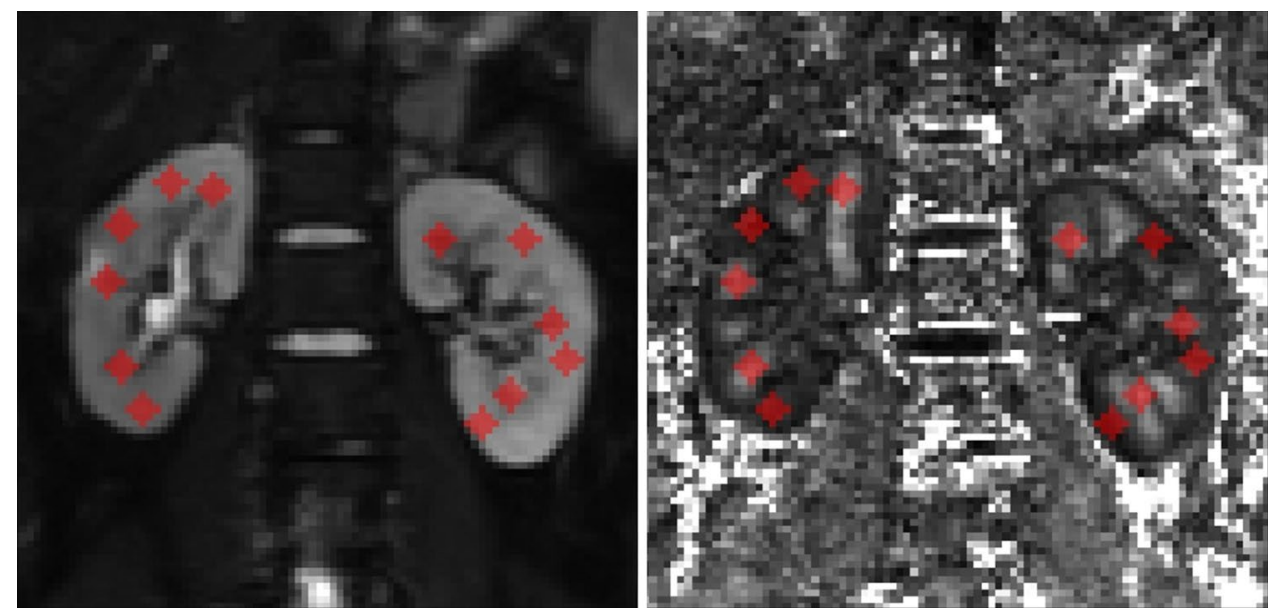

Figure 1. Kidneys regions of interest (ROIs) in a Bardet-Biedl syndrome patient. Baseline, $b=0 \mathrm{~s} / \mathrm{mm}^{2}$ (left) and fractional anisotropy (right) of a BBS subject included in the present study overlaid with ROIs (red circles). The ROIs were positioned in the upper, middle and inferior kidney poles in both the medullary and cortical tissues.

the calculated diffusion tensor. Moreover, the mean image among $b=0 \mathrm{~s} / \mathrm{mm}^{2} \mathrm{DTI}$ images (denoted as b0) was calculated for further processing.

Regions of interest (ROIs) definitions. ITK-SNAP (v. 3.6.0) ${ }^{43}$ was used to annotate ROIs. The ROI generation procedure consisted of manually drawing circular $58 \mathrm{~mm}^{2}$ ROIs at the upper, middle and inferior poles of both cortical and medullary kidney tissues. ROI selection was performed by the radiology technician (L.B.) and confirmed by the radiologist (C.C.). For each subject included in the present study, the ROIs were drawn on coronal slices in the central section of the kidney (i.e. close to the renal hilum) paying attention to avoid the renal pelvis, vessels, calyces and, eventually, renal cysts and fetal lobulations. ROI placement was performed on the b0 image and the corresponding FA map was used to confirm the accuracy of the annotation procedure. The purpose of constraining the ROI placement on a single well-identifiable slice was to create the most reproducible procedure for revealing abnormalities in a diffuse (i.e., non-focal) renal disease, such as BBS. Both right and left kidneys were annotated, resulting in 12 different ROIs for each subject (Fig. 1). Finally, the ROIs were copied to each parametric map.

Statistical analyses. For the following statistical analysis, both cortical and medullary means and standard deviations (SD) from each DTI map were calculated by averaging the ROI values from the upper, middle and inferior poles of each kidney. Moreover, the obtained values were averaged for the left and right kidneys, thereby obtaining a single measurement for both kidneys.

In addition, the CMD derived from each DTI parameter was calculated as the ratio between cortical and medullary mean values. A value close to 1 reflected poor CMD, whereas values farther from 1 represented good differentiation between renal cortical and medullary tissues. By incorporating DTI values evaluated from healthy subjects $^{18,19,21,44}$, FA and AD maps should result in CMD values less than 1 owing to higher FA and AD values in the medulla compared with the renal cortex. Conversely, because MD and RD values reflect the mean diffusivity and the diffusivity parallel to the principal diffusion direction respectively, the CMD of a healthy kidney should result in a value greater than 1.

The statistical analysis was performed using MATLAB r2018a (Mathworks, Inc.).

To investigate renal microstructural injuries in BBS subjects, three specific statistical analyses were performed:

- DTI parameters: for each parametric map, the Wilcoxon rank sum test was performed between mean values of both kidneys, separately for cortical and medullary values, from BBS and control subjects to compare between the two groups.

- CMD analysis: the Wilcoxon rank sum test was applied to CMD values to investigate CMD differences between BBS subjects and controls.

- Kidney asymmetry: the statistical difference between measurements from right and left kidneys within both BBS and control subjects was assessed using the Wilcoxon rank sum test to determine whether DTI parameters of the left kidney significantly differed from those of the right kidney.

For all the statistical tests, $\mathrm{p}<0.05$ was considered statistically significant. 


\begin{tabular}{|l|l|l|l|}
\hline Parameter & BBS & Controls & $\boldsymbol{p}$ Value \\
\hline Age $($ mean \pm SD) & $28 \pm 8$ years & $33 \pm 4$ years & 0.982 \\
\hline Gender $($ female/male) & $5 / 5$ & $4 / 10$ & 0.285 \\
\hline BMI $($ mean \pm SD) & $26.96 \pm 4.78$ & $24.82 \pm 1.48$ & 0.111 \\
\hline eGFR $($ mean \pm SD) & $103.90 \pm 19.34$ & $95.05 \pm 16.01$ & 0.292 \\
\hline
\end{tabular}

Table 1. Main features of Bardet-Biedl syndrome (BBS) patients and controls. BMI: body mass index (Kg/ $\left.\mathrm{m}^{2}\right)$; eGFR: estimated glomerular filtration rate $\left(\mathrm{ml} / \mathrm{min} / 1.73 \mathrm{~m}^{2}\right)$; SD: standard deviation.
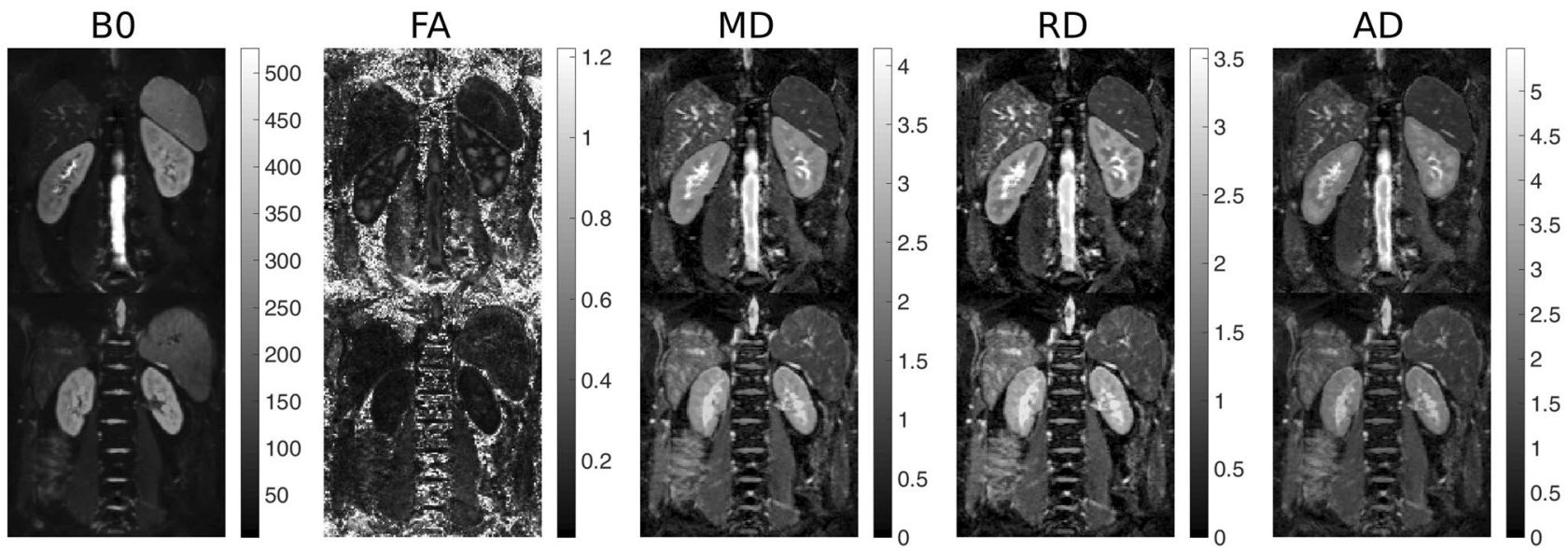

Figure 2. Diffusion Tensor Imaging maps for a Bardet-Biedl syndrome and a control subject. The baseline, $\mathrm{b}=0 \mathrm{~s} / \mathrm{mm}^{2}$ DTI image (B0, left) and derived parametric maps (FA: fractional anisotropy, MD: mean diffusivity, $\mathrm{RD}$ : radial diffusivity and $\mathrm{AD}$ : axial diffusivity, respectively from left to right) for a control (upper) and $\mathrm{BBS}$ subject (lower). The FA images are represented in arbitrary units, whereas the $\mathrm{MD}, \mathrm{RD}$ and $\mathrm{AD}$ images are represented in $10^{-3} \mathrm{~mm}^{2} / \mathrm{s}$.

\section{Results}

Patients cohort. The details of patients and controls are reported in Table 1 . The mean ages were $33 \pm 4$ and $28 \pm 8$ years, respectively, and the eGFRs were in the normal range for both patients and controls, with mean values of $103.90 \pm 19.34$ and $95.05 \pm 16.01 \mathrm{ml} / \mathrm{min} / 1.73 \mathrm{~m}^{2}$, respectively. Additional clinical features of BBS patients included retinal dystrophy in $9 / 10$ patients and learning disabilities were detected in 6 out of 10 BBS individuals. The mean BMI was of $26.96 \mathrm{~kg} / \mathrm{m}^{2}$ in BBS individuals, which was not significantly different from that of the controls. Genetic analysis was available for all the BBS patients, and after a next generation sequencing analysis, $3 / 10$ patients showed no mutations in known BBS-related genes. The remaining patients were homozygous for the BBS4 $(\mathrm{n}=2), B B S 12(\mathrm{n}=2), B B S 1,10$ and $9(\mathrm{n}=1$, each) variants. None of the controls had signs of functional and/or structural renal anomalies.

MRI examinations. After the preliminary visual assessment of image quality, all the acquired images, both from BBS and controls, were suitable for the image analyses procedures. An example of DTI maps, with related CMD values, for a BBS and a control subject included in the present study is shown in Fig. 2.

Renal structural abnormalities were found in six BBS subjects. In particular, three BBS subjects presented renal fetal lobulations (Fig. 3) and four BBS subjects exhibited cysts in the cortical renal tissue with maximum diameter $<6 \mathrm{~mm}$. No morphological kidney abnormalities were found in control subjects.

DTI parameters. The distributions of the medullary and cortical values for both BBS and control subjects are shown in Fig. 4 and the means and SD values from BBS and controls subjects are reported in Table 2. All the DTI parameters from the renal medulla were statistically different between the two groups (Table 2), whereas only cortical FA values differentiated BBS from controls $(p=0.016)$. In particular, medullary FA and AD values were higher in controls than in BBS patients, whereas medullary MD and RD values were higher in BBS subjects than in controls (Table 2).

CMD. The details of the CMD values derived from each DTI parameter for both BBS and control subjects are shown in Table 3. All the CMD values, with the exception of $\mathrm{AD}$, substantially differed between BBS and controls, with the CMD values being higher in controls than BBS subjects. Notably, the mean CMD derived from $\mathrm{MD}$ resulted in an inverted behaviour between $\mathrm{BBS}(\mathrm{CMD}<1)$ and controls $(\mathrm{CMD}>1)$. 

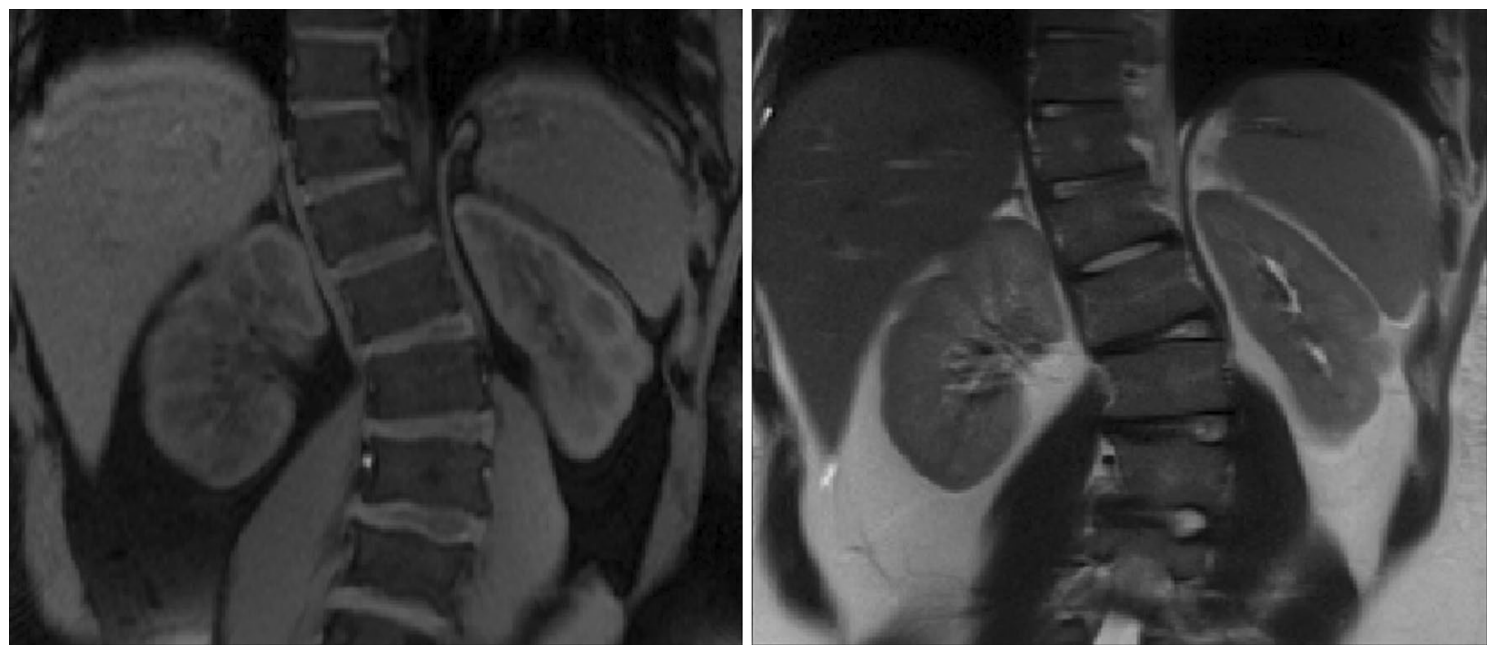

Figure 3. T1- (left) and T2-weighted (right) MRI images of a BBS subject included in the present study. The morphological analysis shows fetal lobulations at the inferior pole of the left kidney.

a
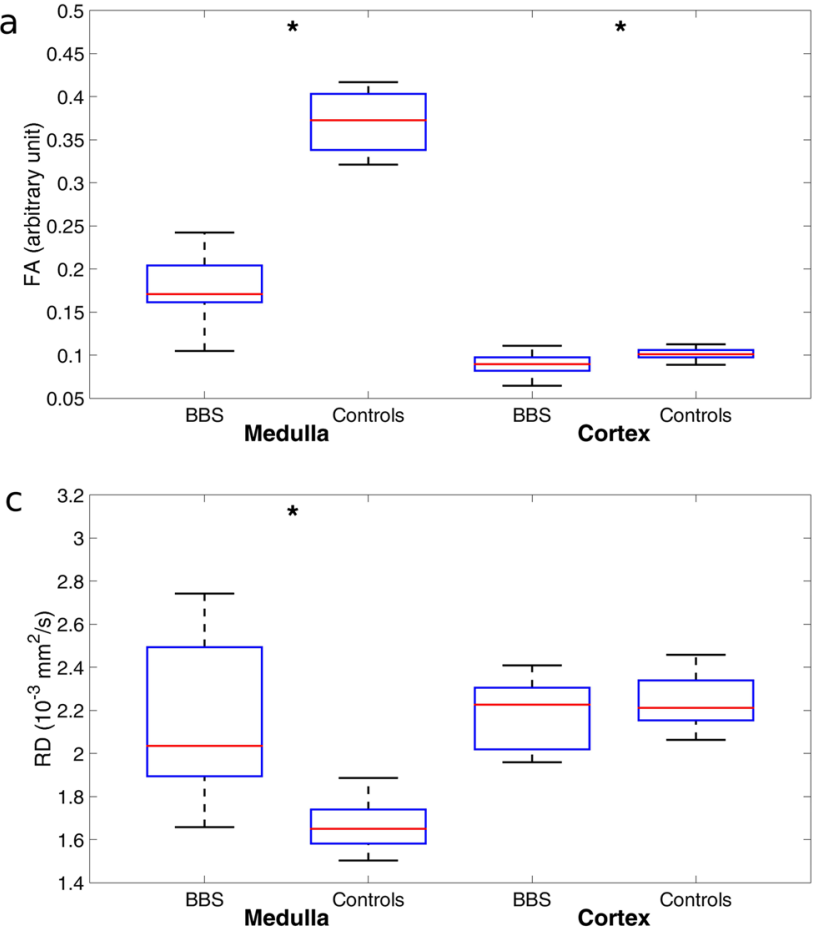

b
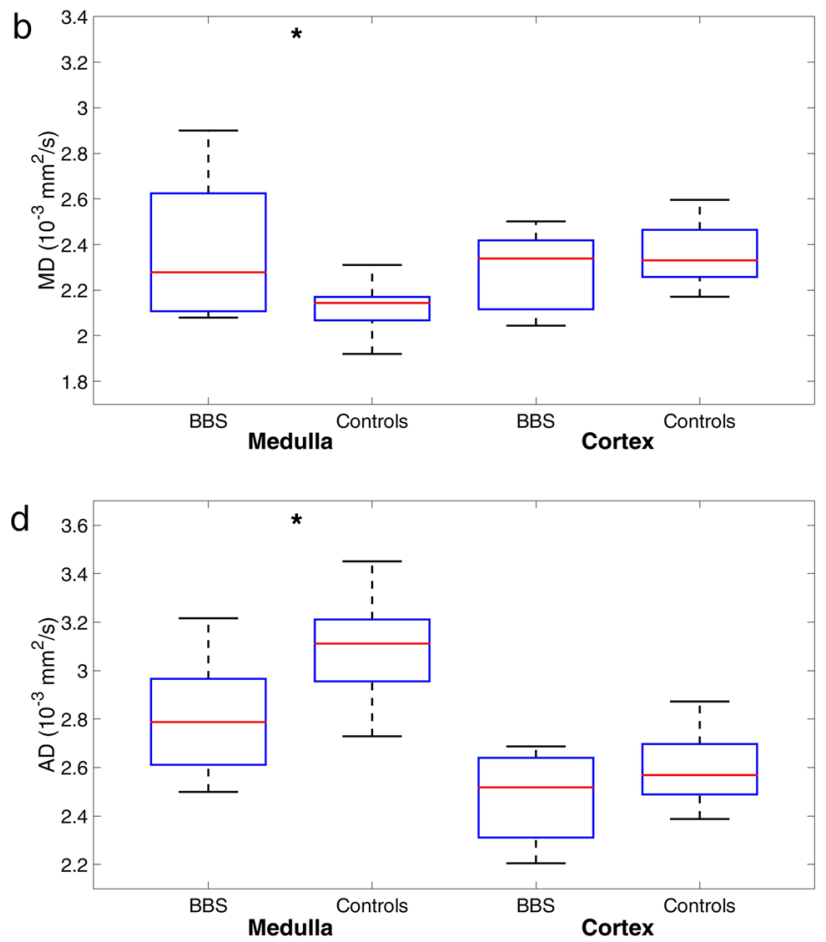

Figure 4. Distribution of both medullary and cortical mean values arising from fractional anisotropy (a), mean diffusivity (b), radial diffusivity (c) and axial diffusivity (d) for BBS and control subjects. Red lines correspond to median values and the blue boxes include from the 25 th to 75 th percentile of the distribution. The asterisks indicate statistically significant differences between BBS subjects and controls.

Kidney asymmetry. Neither BBS subjects nor controls showed statistical differences in DTI parameters from the left and right kidney (Table S1). Moreover, as shown in Table S1, the differences in DTI parameters between groups remained significantly different between patients and controls when the analysis was separately performed for each kidney.

\section{Discussion}

This is the first study using DTI to characterise renal abnormalities in BBS patients before the onset of clinically evident renal dysfunction. The latter is an important cause of morbidity and mortality in BBS patients and, to date, the exact pathomechanisms leading to kidney disease in these patients is unknown. Moreover, because $\mathrm{CKD}$ in BBS, as well as in the general population, is a progressive disorder characterised by a time-dependent 


\begin{tabular}{|l|l|l|r|}
\hline & BBS & Controls & $\boldsymbol{p}$ Value \\
\hline Cortical FA & $0.089[0.014]$ & $0.101[0.008]$ & $\mathbf{0 . 0 3 3}$ \\
\hline Medullary FA & $0.171[0.034]$ & $0.373[0.058]$ & $<\mathbf{0 . 0 0 1}$ \\
\hline Cortical MD & $2.339[0.271]$ & $2.330[0.190]$ & 0.429 \\
\hline Medullary MD & $2.278[0.415]$ & $2.144[0.095]$ & $\mathbf{0 . 0 4 3}$ \\
\hline Cortical RD & $2.227[0.262]$ & $2.212[0.171]$ & 0.501 \\
\hline Medullary RD & $2.035[0.474]$ & $1.650[0.140]$ & $<\mathbf{0 . 0 0 1}$ \\
\hline Cortical AD & $2.518[0.308]$ & $2.568[0.197]$ & 0.151 \\
\hline Medullary AD & $2.788[0.329]$ & $3.111[0.236]$ & $\mathbf{0 . 0 2 1}$ \\
\hline
\end{tabular}

Table 2. Bardet-Biedl syndrome (BBS) and controls median and interquartile ranges (in square brackets) for DTI parameters from both the cortical and medullary regions. FA: fractional anisotropy; MD: mean diffusivity; RD: radial diffusivity; $\mathrm{AD}$ : axial diffusivity. FA values are expressed in arbitrary units, whereas $\mathrm{MD}, \mathrm{RD}$ and $\mathrm{AD}$ values are reported in $10^{-3} \mathrm{~mm}^{2} / \mathrm{s}$. p-values $<0.05$ are represented in bold.

\begin{tabular}{|l|l|l|r|}
\hline & BBS & Controls & p-value \\
\hline FA & $0.546[0.141]$ & $0.268[0.045]$ & $<\mathbf{0 . 0 0 1}$ \\
\hline MD & $0.956[0.053]$ & $1.100[0.063]$ & $<\mathbf{0 . 0 0 1}$ \\
\hline RD & $0.992[0.080]$ & $1.350[0.072]$ & $<\mathbf{0 . 0 0 1}$ \\
\hline AD & $0.889[0.074]$ & $0.828[0.063]$ & 0.063 \\
\hline
\end{tabular}

Table 3. Corticomedullary differentiation, reported as median and interquartile ranges (in square brackets), which were calculated as the ratios between cortical and medullary mean values for each DTI parameter. FA: fractional anisotropy; MD: mean diffusivity; RD: radial diffusivity; AD: axial diffusivity; BBS: Bardet-Biedl syndrome. p-values $<0.05$ are presented in bold.

progression, the early diagnosis of kidney abnormalities will have great clinical impact, because it allows the determination of patients at risk of progressing renal disease.

To date, limited information is available in the literature on markers of early CKD in BBS patients. As in nonsyndromic individuals, an increased plasma creatinine levels is not an early index of kidney dysfunction. This study demonstrates that the major parameters used to address renal dysfunction are sometimes not sufficient to detect small kidney defects that may predispose a patient to CKD progression ${ }^{31}$. For this purpose, we selected BBS patients with eGFR values greater than $60 \mathrm{ml} / \mathrm{min} / 1.73 \mathrm{~m}^{2}$. This subgroup of individuals included young adult subjects with plasma creatinine level in the normal range. Moreover, consistent with the well-documented theory that kidney disease in this setting is not primarily a glomerular disorder, these patients did not show significant increases in urine protein excretion. Thus, the BBS patients we selected did not show alterations in the major parameters used in the clinical setting to address renal dysfunction.

As a primary finding, DTI was able to discriminate BBS patients from age-gender-eGFR-BMI matched controls. Indeed, DTI values differed between BBS and controls, especially in the renal medulla, in which more tubular structures are represented. The altered values in the medullary renal tissues of BBS subjects may reflect the compromised integrity of collecting ducts as a sign of early tubulointerstitial injuries, which is a common feature that causes urine concentration dysfunction in $\mathrm{BBS}^{45}$. Among the characteristics, the FA was able to best discriminate BBS from controls because it measures the preferential directionality of water molecules within tissues. In this setting, alterations in FA values may reflect microstructural changes in the kidneys of BBS subjects. Our findings are in-line with a recent study in which FA values identified early renal damage in diabetic patients with normal BMI and eGFR values ${ }^{31}$.

Moreover, poorer CMD was revealed in BBS patients, indicating that the CMD may represent an additional index of early renal abnormalities in BBS. The reduction of CMD in BBS patients was likely owing to the significant alterations in the DTI values of medullary tissues compared with the controls. Therefore, the kidney injuries measured using DTI were not specifically related to the left or right kidney, which confirmed the symmetry of CKD-related renal damage in BBS patients ${ }^{3}$.

The primary limitation of the present study is the small sample size. However, the limited number of subjects is due to the rarity of BBS and the presence of constraints on MRI examinations (i.e., BMI). As a second limitation, no direct comparison of the presented findings were performed with DTI parameters obtained from subjects with other kidney structural abnormalities (such as congenital renal dysplasia). In addition, because the morbidly obese cannot undergo MRI studies, the analysis was applied to mainly overweight BBS patients with no severe obesity. In this scenario, obesity may confound the assessment of kidney diffusion parameters based on the $\mathrm{DTI}^{46}$. Finally, the genetic variability in the BBS subjects, together with the small sample size, made it difficult to assess genotype to phenotype correlations. Further studies with larger numbers of BBS subjects may untangle possible associations between specific BBS gene mutations and kidney phenotypes using the DTI technique. 
It is worth underlining the importance of the non-invasiveness of renal DTI. Indeed, as specified in the methodological section, the DTI technique does not require the use of any contrast media and, therefore, is suitable for renal pathologies, thus deserving attention for the translation in clinical practice.

In conclusion, this study confirmed the ability of the DTI technique to reveal renal abnormalities before the clinical onset of renal dysfunction, demonstrating its suitability as a tool for detecting early renal injuries.

\section{Data availability}

MR data can be made available under request to the corresponding author (pending the approval of the Research Ethics Board).

Received: 25 June 2021; Accepted: 11 October 2021

Published online: 21 October 2021

\section{References}

1. Zacchia, M., Capolongo, G., Trepiccione, F. \& Marion, V. Impact of local and systemic factors on kidney dysfunction in bardetbiedl syndrome. Kidney Blood Press Res. 42, 784-793 (2017).

2. Zacchia, M. et al. Proteomics and metabolomics studies exploring the pathophysiology of renal dysfunction in autosomal dominant polycystic kidney disease and other ciliopathies. Nephrol. Dial. Transplant. 35, 1853-1861 (2020).

3. Forsythe, E. et al. Risk factors for severe renal disease in bardet-biedl syndrome. JASN 28, 963-970 (2017).

4. Zacchia, M. et al. Urine concentrating defect as presenting sign of progressive renal failure in Bardet-Biedl syndrome patients. Clin. Kidney J. 14, 1545-1551 (2021).

5. Marchese, E., Ruoppolo, M., Perna, A., Capasso, G. \& Zacchia, M. Exploring key challenges of understanding the pathogenesis of kidney disease in Bardet-Biedl syndrome. Kidney Int. Rep. 5, 1403-1415 (2020).

6. Zacchia, M. et al. Renal phenotype in Bardet-Biedl syndrome: a combined defect of urinary concentration and dilution is associated with defective urinary AQP2 and UMOD excretion. Am. J. Physiol. Renal Physiol. 311, F686-F694 (2016).

7. Esposito, G. et al. Genetic characterization of Italian patients with Bardet-Biedl syndrome and correlation to ocular, renal and audio-vestibular phenotype: identification of eleven novel pathogenic sequence variants. BMC Med Genet 18, 10 (2017).

8. Zhang, W., Blumenfeld, J. D. \& Prince, M. R. MRI in autosomal dominant polycystic kidney disease. J. Magn. Reson. Imaging 50, 41-51 (2019).

9. Cornelis, F. et al. Multiparametric magnetic resonance imaging for the differentiation of low and high grade clear cell renal carcinoma. Eur Radiol 25, 24-31 (2015).

10. Zhang, H. et al. High signal renal tumors on DWI: the diagnostic value of morphological characteristics. Abdom Radiol 44, 239-246 (2019).

11. Li, L.-P. et al. Medullary blood oxygen level-dependent MRI index (R2*) is associated with annual loss of kidney function in moderate CKD. AJN 51, 966-974 (2020).

12. Morrell, G. R., Zhang, J. L. \& Lee, V. S. Magnetic resonance imaging of the fibrotic kidney. JASN 28, 2564-2570 (2017).

13. Prasad, P. V. et al. Cortical perfusion and tubular function as evaluated by magnetic resonance imaging correlates with annual loss in renal function in moderate chronic kidney disease. AJN 49, 114-124 (2019).

14. Selby, N. M. et al. Magnetic resonance imaging biomarkers for chronic kidney disease: a position paper from the European Cooperation in Science and Technology Action PARENCHIMA. Nephrology Dialysis Transplantation 33, ii4-ii14 (2018).

15. Simms, R. \& Sourbron, S. Recent findings on the clinical utility of renal magnetic resonance imaging biomarkers. Nephrol. Dial. Transplant. 35, 915-919 (2020).

16. Srivastava, A. et al. Kidney functional magnetic resonance imaging and change in eGFR in individuals with CKD. CJASN 15, 776-783 (2020)

17. Notohamiprodjo, M., Reiser, M. F. \& Sourbron, S. P. Diffusion and perfusion of the kidney. Eur. J. Radiol. 76, 337-347 (2010)

18. Kataoka, M. et al. Diffusion tensor imaging of kidneys with respiratory triggering: optimization of parameters to demonstrate anisotropic structures on fraction anisotropy maps. J. Magn. Reson. Imaging 29, 736-744 (2009).

19. Ries, M., Jones, R. A., Basseau, F., Moonen, C. T. W. \& Grenier, N. Diffusion tensor MRI of the human kidney. J. Magn. Reson. Imaging 14, 42-49 (2001).

20. Gaudiano, C. et al. Renal diffusion tensor imaging: is it possible to define the tubular pathway? A case report. Magnetic Resonance Imaging 29, 1030-1033 (2011).

21. Gurses, B., Kilickesmez, O., Tasdelen, N., Firat, Z. \& Gurmen, N. Diffusion tensor imaging of the kidney at 3 tesla: normative values and repeatability of measurements in healthy volunteers. Diagn. Interv. Radiol. https://doi.org/10.4261/1305-3825.DIR.3892-10.1 (2010).

22. Wang, W. et al. MR diffusion tensor imaging of normal kidneys: DTI of Normal Kidneys at 3T. J. Magn. Reson. Imaging 40, 1099-1102 (2014).

23. Gaudiano, C. et al. Diffusion tensor imaging and tractography of the kidneys: assessment of chronic parenchymal diseases. Eur. Radiol. 23, 1678-1685 (2013).

24. Liu, Z. et al. Chronic kidney disease: pathological and functional assessment with diffusion tensor imaging at 3T MR. Eur. Radiol. 25, 652-660 (2015).

25. Wang, W. et al. 3T magnetic resonance diffusion tensor imaging in chronic kidney disease. Abdom. Imaging 39, 770-775 (2014).

26. Deger, E. et al. Rejection evaluation after renal transplantation using MR diffusion tensor imaging. Acta Radiol 59, 876-883 (2018).

27. Fan, W. et al. Assessment of renal allograft function early after transplantation with isotropic resolution diffusion tensor imaging. Eur. Radiol. 26, 567-575 (2016).

28. Palmucci, S. et al. Diffusion weighted imaging and diffusion tensor imaging in the evaluation of transplanted kidneys. Eur. J. Radiol. Open 2, 71-80 (2015).

29. Razek, A. A. K. A., Al-Adlany, M. A. A. A., Alhadidy, A. M., Atwa, M. A. \& Abdou, N. E. A. Diffusion tensor imaging of the renal cortex in diabetic patients: correlation with urinary and serum biomarkers. Abdominal Radiol. 42, 1493-1500 (2017).

30. Wang, Y.-C., Feng, Y., Lu, C.-Q. \& Ju, S. Renal fat fraction and diffusion tensor imaging in patients with early-stage diabetic nephropathy. Eur. Radiol. 28, 3326-3334 (2018).

31. Ye, X. J. et al. Using magnetic resonance diffusion tensor imaging to evaluate renal function changes in diabetic patients with early-stage chronic kidney disease. Clin. Radiol. 74, 116-122 (2019).

32. Feng, Q. et al. Renal clear cell carcinoma: diffusion tensor imaging diagnostic accuracy and correlations with clinical and histopathological factors. Clin. Radiol. 72, 560-564 (2017).

33. Feng, Q., Ma, Z., Wu, J. \& Fang, W. DTI for the assessment of disease stage in patients with glomerulonephritis - correlation with renal histology. Eur. Radiol. 25, 92-98 (2015).

34. Marion, V. et al. Bardet-Biedl syndrome highlights the major role of the primary cilium in efficient water reabsorption. Kidney Int. 79, 1013-1025 (2011). 
35. Imhoff, O. et al. Bardet-Biedl syndrome: a study of the renal and cardiovascular phenotypes in a french cohort. CJASN 6, 22-29 (2011).

36. Beales, P. L., Elcioglu, N., Woolf, A. S., Parker, D. \& Flinter, F. A. New criteria for improved diagnosis of Bardet-Biedl syndrome: results of a population survey. J Med Genet 36, 437-446 (1999).

37. Cutajar, M., Clayden, J. D., Clark, C. A. \& Gordon, I. Test-retest reliability and repeatability of renal diffusion tensor MRI in healthy subjects. Eur. J. Radiol. 80, e263-e268 (2011).

38. Wang, W. et al. MR diffusion tensor imaging of normal kidneys. J. Magn. Reson. Imaging 40, 1099-1102 (2014).

39. Borrelli, P. et al. Diffusion tensor imaging of the kidney: design and evaluation of a reliable processing pipeline. Sci Rep $\mathbf{9}, 12789$ (2019).

40. Smith, S. M. et al. Advances in functional and structural MR image analysis and implementation as FSL. Neuroimage 23, S208-S219 (2004).

41. Andersson, J. L. R., Skare, S. \& Ashburner, J. How to correct susceptibility distortions in spin-echo echo-planar images: application to diffusion tensor imaging. Neuroimage 20, 870-888 (2003).

42. Tournier, J.-D. et al. MRtrix3: A fast, flexible and open software framework for medical image processing and visualisation. NeuroImage 202, 116137 (2019).

43. Yushkevich, P. A. et al. User-guided 3D active contour segmentation of anatomical structures: significantly improved efficiency and reliability. Neuroimage 31, 1116-1128 (2006).

44. Notohamiprodjo, M. et al. Diffusion tensor imaging of the kidney with parallel imaging: initial clinical experience. Invest. Radiol. 43, 677-685 (2008).

45. Caterino, M. et al. Urine proteomics revealed a significant correlation between urine-fibronectin abundance and estimated-GFR decline in patients with Bardet-Biedl syndrome. Kidney Blood Press Res. 43, 389-405 (2018).

46. Ebrahimi, B. et al. Renal adiposity confounds quantitative assessment of markers of renal diffusion with MRI: a proposed correction method. Invest. Radiol. 52(11), 672 (2017).

\section{Acknowledgements}

This work has been partially funded by italian Ministry of Health ("Ricerca corrente" project). This work is generated within the European Reference Network for Rare Kidney Diseases (ERKNet).

\section{Author contributions}

P.B. and M.Z. designed the study; P.B. M.A. and L.B. performed the data analysis; C.C., L.B. data acquisition and radiological evaluation; P.B., and M.A. conducted the statistical analysis; P.B. and M.Z. wrote the paper; G.C., M.S. and M.A. supervised the procedures and revised the manuscript. All the authors reviewed the manuscript.

\section{Competing interests}

The authors declare no competing interests.

\section{Additional information}

Supplementary Information The online version contains supplementary material available at https:/doi.org/ 10.1038/s41598-021-00394-4.

Correspondence and requests for materials should be addressed to P.B.

Reprints and permissions information is available at www.nature.com/reprints.

Publisher's note Springer Nature remains neutral with regard to jurisdictional claims in published maps and institutional affiliations.

(c) Open Access This article is licensed under a Creative Commons Attribution 4.0 International (c) License, which permits use, sharing, adaptation, distribution and reproduction in any medium or format, as long as you give appropriate credit to the original author(s) and the source, provide a link to the Creative Commons licence, and indicate if changes were made. The images or other third party material in this article are included in the article's Creative Commons licence, unless indicated otherwise in a credit line to the material. If material is not included in the article's Creative Commons licence and your intended use is not permitted by statutory regulation or exceeds the permitted use, you will need to obtain permission directly from the copyright holder. To view a copy of this licence, visit http://creativecommons.org/licenses/by/4.0/.

(C) The Author(s) 2021 